\title{
Quantitative reconstruction of a magnetic nanoparticle distribution using a non-negativity constraint
}

\author{
Maik Liebl $^{1}$, Uwe Steinhoff ${ }^{1}$, Frank Wiekhorst ${ }^{1}$, Annelies Coene ${ }^{2}$, Jens Haueisen ${ }^{3}$, Lutz Trahms ${ }^{1}$ \\ ${ }^{1}$ Physikalisch-Technische Bundesanstalt, 10587 Berlin, Germany \\ ${ }^{2}$ Department of Electrical Energy, Systems and Automation, Ghent University, 9000 Ghent, Belgium \\ ${ }^{3}$ Institute of Biomedical Engineering and Informatics, Ilmenau University of Technology, 98693 Ilmenau, \\ Germany \\ E-Mail: maik.liebl@ptb.de
}

\begin{abstract}
Magnetorelaxometry (MRX) is a non-invasive method for the specific quantification of magnetic nanoparticles (MNP). Here, we investigate experimentally the reconstruction of the MNP concentration in an extended volume. A phantom with varying but known MNP distribution was subsequently magnetized by 48 planar coils at different locations. The MRX signal was measured using the PTB 304 SQUID-magnetometer system. The inverse problem was solved by means of a nonnegative least squares (NNLS) algorithm and compared to a minimum norm estimation (TSVD-MNE). The reconstruction by NNLS shows a deviation of the total MNP amount of less than $3 \%(10 \%$ by TSVD-MNE). Hence, adapted non-invasive MRX methods can reliable reconstruct the MNP content in extended volumes.
\end{abstract}

Keywords: Magnetorelaxometry, magnetic nanoparticles, inhomogeneous magnetizing fields, spatial encoding

\section{Introduction}

Magnetic nanoparticles (MNP) are promising tools for novel cancer treatment approaches [1], e.g. as thermal actuators in magnetic hyperthermia or as nanovehicles to deliver drugs in magnetic drug targeting. An essential prerequisite for the development of these approaches is a quantitative knowledge of the MNP distribution inside a body. MNP exposed to sudden changes of an external magnetic field exhibit a characteristic magnetic response that is utilized in magnetorelaxometry (MRX). MRX allows the non-invasive quantification of the MNP content inside biological samples [2]. Using a multi-sensor device a spatially resolved quantification of multiple MNP accumulations becomes feasible [2,3]. Furthermore, by sequentially applying different inhomogeneous excitation fields a spatial encoding of the MNP in a sample was achieved that increased the spatial resolution of the reconstruction, as suggested theoretically in $[4,5,6]$. Since each excitation field entails a set of up to $N$ (=number of sensors of the MRX device) linear independent equations in the forward problem, the inverse problem can be stabilized. So far the reconstruction was accomplished by means of minimum-norm estimation using a truncated singular value decomposition (denoted as TSVD-MNE) $[5,6]$, potentially resulting in negative estimates that are physically non-plausible (negative values of MNP content). In this paper we investigate the use of a nonnegative least squares (NNLS) algorithm [7] for the reconstruction of an MNP distribution from experimental MRX data.

\section{Methods}

MRX measurements after inhomogeneous magnetization The experimental setup is sketched in fig. 1 .

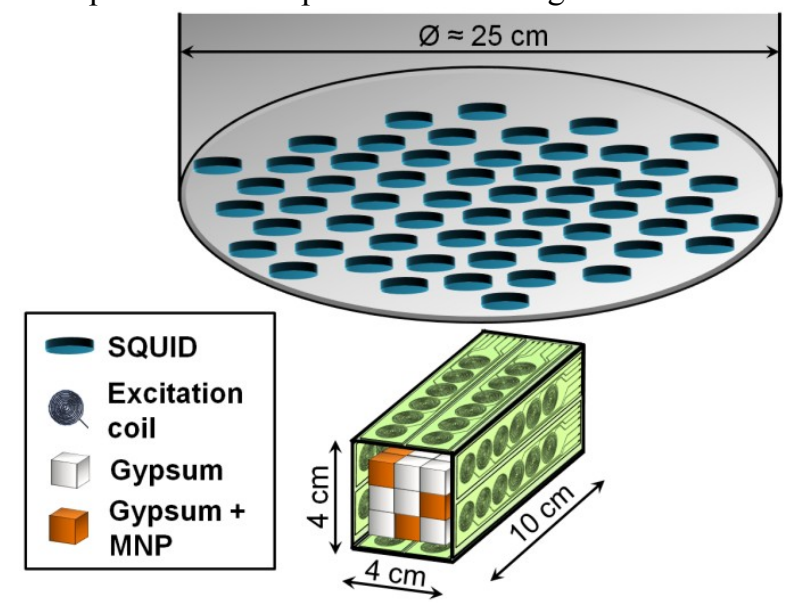

Figure 1: Sketch of the measurement setup used for MRX with inhomogeneous magnetizing of the volume phantom showing the 304 SQUID device, arrangement of magnetizing coils and 54 gypsum cubes (12 MNP loaded).

We used a compact volume phantom assembled of 54 individual gypsum cubes of $1 \mathrm{~cm}^{3}$ volume. Into 12 of the cubes a certain MNP amount (Berlin Heart GmbH, Berlin, Germany) was blended during fabrication and estimated by homogeneous MRX according to [2]. The estimated MNP amount of the single cubes was $5 \pm 0.07 \mathrm{mg}$. The cubes were assembled to form a nominal MNP distribution as shown in fig 2. Alongside the phantom 48 planar spiral coils were mounted providing inhomogeneous magnetizing fields with different spatial configuration. Each coil was fed by a current of $600 \mathrm{~mA}$ for a $1 \mathrm{~s}$ time interval. After switching off an individual coil and a 450 $\mu$ s delay time the relaxation signals $\mathbf{B}(t)$ of the 171 sensors were recorded for $2 \mathrm{~s}$ at a sampling rate of $250 \mathrm{~Hz}$. To improve the signal-to-noise ratio (SNR) five measurements were averaged for each coil. The relaxation amplitudes $\Delta B_{\text {meas }}$ were calculated as the difference of the averaged relaxation signals between two fixed time instants

$$
\Delta B_{\text {meas }}=B_{t_{1}=100 m s}-B_{t_{2}=1.5 \mathrm{~s}}
$$


The forward problem

We divide the sample volume into $K$ voxels and aim to determine the MNP amounts $X_{\mathrm{MNP}, k}$ of each voxel (assuming a uniform MNP distribution within each voxel). In case of small magnetizing fields (below $1 \mathrm{mT}$ ) the partial magnetic flux density $B_{k}$ at the sensor location $\mathbf{r}_{\mathrm{s}}$ resulting from the relaxation of MNP within the $k$ th voxel is proportional to $\chi_{\mathrm{MRX}} \cdot \mathbf{H}_{k} \cdot X_{\mathrm{MNP}, k}$. The relaxation susceptibility $\chi_{\mathrm{MRX}}$ (relaxation moment for given magnetizing and relaxation intervals normalized to MNP mass and unit magnetizing field) is MNP specific and was determined separately by a conventional MRX measurement with homogeneous magnetizing of a single MNP containing cube. Thus the magnetic flux density is given by

$\Delta B_{k}=\frac{\mu_{0}}{4 \pi}\left\lfloor\left(\frac{3 \cdot \mathbf{n}^{\mathrm{T}}\left(\mathbf{r}_{k} \mathbf{r}_{k}^{\mathrm{T}}\right)}{\left|\mathbf{r}_{k}\right|^{5}}-\frac{\mathbf{n}^{\mathrm{T}}}{\left|\mathbf{r}_{k}\right|^{3}}\right) \cdot \mathbf{H}_{k} \chi_{\mathrm{MRX}}\right\rfloor X_{\mathrm{MNP}, k}$

with $\mathbf{n}$ being the normal vector of the sensor orientation and $\mathbf{H}_{k}$ the magnetizing field in the $k$ th voxel. The right hand side of eq. 2 can be separated into geometry parameters and source parameters to $\Delta B_{k}=\mathbf{L} \cdot X_{\mathrm{MNP}, k}$. The total relaxation amplitude $\Delta B$ follows by superposition of the field contributions of all voxels

$$
\Delta B=\sum_{k} L_{k} X_{M N P, k}=\mathbf{L} \cdot \mathbf{X}_{M N P}
$$

Using multiple magnetizing coils and multiple sensors, $\Delta \mathbf{B}$ becomes a vector and $\mathbf{L}$ is a matrix of dimension $\left(N_{\text {sensors }} \cdot N_{\text {coils }} \times K_{\text {voxels }}\right)$.

\section{The inverse problem}

Using the vector $\Delta \mathbf{B}_{\text {meas }}$ containing the measured relaxation amplitudes the general least square problem can be formulated as

$$
\Delta^{2}=\min \left\|\mathbf{L} \cdot \mathbf{X}_{M N P}-\Delta \mathbf{B}_{\text {meas }}\right\|^{2}
$$

where the difference between the forward solution and $\Delta \mathbf{B}_{\text {meas }}$ has to be minimized. In [5,6] this was done by a minimum norm estimation employing the Moore-Penrose pseudoinverse $\mathbf{L}^{+}=\left(\mathbf{L}^{\mathrm{T}} \mathbf{L}\right)^{-1} \mathbf{L}^{\mathrm{T}}$ calculated by a TSVD

$$
\hat{\mathbf{X}}_{M N P}=\mathbf{L}^{+} \Delta \mathbf{B}
$$

Alternatively, eq. 4 can be also solved by an active set NNLS algorithm [7] until a set of $\widehat{\mathbf{X}}_{\mathrm{MNP}}$ is found that satisfies the constraints $\widehat{\mathbf{X}}_{\mathrm{MNP}} \geq 0$. In order to avoid use of a priori on the location of the cubes, the reconstruction grid describing voxel positions $\mathbf{r}_{\mathrm{v}}$ was shifted in x-direction out of the volume center and resampled from the real $\left(3 N_{\mathrm{x}} \cdot 6 N_{\mathrm{y}} \cdot 3 N_{\mathrm{z}}\right)$ grid towards a $4 N_{\mathrm{x}} \cdot 6 N_{\mathrm{y}} \cdot 3 N_{\mathrm{z}}$ grid as sketched in fig. 2 .

\section{Results}

The results obtained by both estimations are shown together with the nominal MNP distribution in fig 2. For both algorithms the reconstructed total MNP amount is close to the nominal amount of MNP. The TSVD-MNE reconstructed in some voxels negative MNP amounts and resulted in total MNP content that was about $10 \%$ below the nominal value. As expected, less deviation (3\%) was found for the NNLS reconstruction.

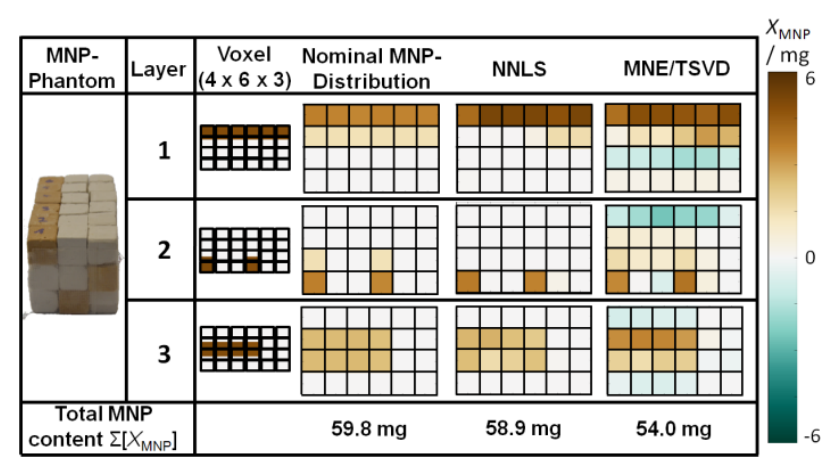

Figure 2: Nominal MNP cube distribution over each of the 3 layers of the phantom and reconstructed MNP amounts by NNLS and TSVD-MNE. The total MNP amount of the phantom is given in the bottom line.

\section{Discussion}

We investigated the quantitative imaging of an MNP distribution using sequential MRX with inhomogeneous magnetization $[5,6]$. We demonstrated that it is experimentally possible to reconstruct distributed MNP content in the milligram $/ \mathrm{cm}^{3}$ range within a volume of $54 \mathrm{~cm}^{3}$. We also showed that the NNLS method can improve the quality of the reconstruction.

\section{Acknowledgement}

This work was financially supported by the DFG research programs "Magnetische Nanopartikel für die Krebstherapie" TR408/4-3 and "Magnetische Nanopartikel für die Zelluläre und Molekulare MR-Bildgebung” TR408/5-2.

\section{Bibliography}

[1] Q. A. Pankhurst et. al.: Applications of magnetic nanoparticles in biomedicine, J. Phys. D: Appl. Phys., vol. 36, pp. R167-R181, 2003.

[2] F. Wiekhorst et al.: Magnetorelaxometry Assisting Biomedical Applications of Magnetic Nanoparticles, Pharm. Res., vol. 29(5), pp. 1189-202, 2012.

[3] D. Baumgarten et al.: Magnetic nanoparticle imaging by means of minimum norm estimates from remanence measurements, Med. Biol. Eng. Comp., vol. 46, pp. 1177-1185, 2008.

[4] N. G. Sepulveda et al.: Magnetic susceptibility tomography for three dimensional imaging of diamagnetic and paramagnetic objects, IEEE Trans. Magn., vol. 30, pp. 5062-5069, 1994.

[5] U. Steinhoff et al.: Bildgebung magnetischer Nanopartikel basierend auf Magnetorelaxometrie mit sequentieller Aktivierung inhomogener Anregungsfelder, Biomed. Tech., vol. 55(1), pp. 22-25, 2010.

[6] G. Crevecoeur et al.: Advancements in magnetic nanoparticle reconstruction using sequential activation of excitation coil arrays using magnetorelaxometry, IEEE Trans. on Magn., vol. 48, pp. 1313-1316, 2012

[7] C. L. Lawson et al.: Solving Least Squares Problems, Prentice Hall., Chapter 23, p. 161, 1974. 